\title{
Emergence of Sensory Patterns during Sleep Highlights Differential Dynamics of REM and Non-REM Sleep Stages
}

\author{
Michal Ramot, ${ }^{1}$ Lior Fisch, ${ }^{2}$ Ido Davidesco, ${ }^{1}$ Michal Harel,, 2 Svetlana Kipervasser, ${ }^{3,5}$ Fani Andelman, ${ }^{4}$ Miri Y. Neufeld, ${ }^{3,5}$ \\ Uri Kramer, ${ }^{3,5}$ Itzhak Fried, ${ }^{4,6}$ and Rafael Malach ${ }^{2}$ \\ ${ }^{1}$ Interdisciplinary Center for Neural Computation, The Hebrew University of Jerusalem, Jerusalem 91904, Israel, ${ }^{2}$ Department of Neurobiology, Weizmann \\ Institute of Science, Rehovot 76100, Israel, ${ }^{3} \mathrm{EEG}$ and Epilepsy Unit, Department of Neurology, and ${ }^{4}$ Functional Neurosurgery Unit, Tel Aviv Sourasky \\ Medical Center, Tel Aviv 64239, Israel, ${ }^{5}$ Sackler School of Medicine, Tel Aviv University, Tel Aviv 69978, Israel, and ${ }^{6}$ Department of Neurosurgery, David \\ Geffen School of Medicine, University of California at Los Angeles, Los Angeles, California 90095
}

Despite the profound reduction in conscious awareness associated with sleep, sensory cortex remains highly active during the different sleep stages, exhibiting complex interactions between different cortical sites. The potential functional significance of such spatial patterns and how they change between different sleep stages is presently unknown. In this electrocorticography study of human patients, we examined this question by studying spatial patterns of activity (broadband gamma power) that emerge during sleep (sleep patterns) and comparing them to the functional organization of sensory cortex that is activated by naturalistic stimuli during the awake state. Our results show a high correlation $\left(p<10^{-4}\right.$, permutation test) between the sleep spatial patterns and the functional organization found during wakefulness. Examining how the sleep patterns changed through the night highlighted a stage-specific difference, whereby the repertoire of such patterns was significantly larger during rapid eye movement (REM) sleep compared with non-REM stages. These results reveal that intricate spatial patterns of sensory functional organization emerge in a stage-specific manner during sleep.

\section{Introduction}

Sleep presents a particularly intriguing challenge to brain research. On the one hand, it entails (at least in non-dream states) a massive loss of consciousness. On the other hand, the brain, and particularly the cerebral cortex, is far from being silent during such nonconscious states. Thus, a large body of research has revealed that during different stages of sleep cortical networks are highly active- - both at the level of single neuron activity and in large neuronal assemblies (Steriade et al., 1993, 2001; Contreras and Steriade, 1995; Crunelli and Hughes, 2010). It has been shown that the sleep activity patterns are not random or chaotic (Volgushev et al., 2006; Chauvette et al., 2010; Andrillon et al., 2011; Nir et al., 2011), and there have been hints that they may be influenced by prior experience (Huber et al., 2004, 2006; Esser et al., 2006). However, we are still largely in the dark with regard to the meaning or information content reflected in the spatial organization and dynamic modulations of these patterns of activity.

A particularly relevant study using voltage-sensitive dye imaging (Kenet et al., 2003) has revealed that spatial sleep patterns (correlated activations of cortical sites) observed in the primary

Received Jan. 17, 2013; revised July 6, 2013; accepted July 25, 2013.

Author contributions: M.R. and R.M. designed research; M.R., L.F., I.D., S.K., F.A., M.Y.N., U.K. and I.F. performed research; M.R., L.F., and M.H. analyzed data; M.R. and R.M. wrote the paper.

This study was funded by the Kimmel Award for Innovative Research and a Bikura grant from the Israel Science Foundation to R.M., and by a grant from the WIS-Ichilov Fund to R.M. and I.F. We thank the participants for volunteering to take part in the study, as well as D. Yossef, S. Nagar, R. Cohen, C. Yosef, G. Yehezkel, and the EEG technicians for assistance at the Tel Aviv Medical Center.

Correspondence should be addressed to Rafael Malach, Department of Neurobiology, Weizmann Institute of Science, Rehovot 76100, Israel. E-mail: Rafi.Malach@weizmann.ac.il

DOI:10.1523/JNEUROSCI.0232-13.2013

Copyright $\odot 2013$ the authors $\quad 0270-6474 / 13 / 3314715-14 \$ 15.00 / 0$ visual cortex of anesthetized cats correspond to the well known functional organization of orientation or ocular dominance columns. This finding raises the intriguing possibility that the patterns that emerge during natural sleep in the human sensory cortex may also reflect the underlying functional organization structure of sensory networks. A hint that such a phenomenon may indeed occur in the human cortex was provided recently in the finding of a high level of interhemispheric activity correlation between homologous cortical points in human sensory cortex during sleep (He et al., 2008; Nir et al., 2008; Larson-Prior et al., 2011). However, since no sensory-driven information was available for these studies, the similarity of these correlation patterns to the functional organization of sensory networks remained unknown.

Above and beyond the averaged patterns, the possibility of moment-by-moment changes in the sleep patterns are of particular interest, especially with regard to possible differences between rapid eye movement (REM) and non-REM (NREM) sleep stages. REM sleep has a very different electrophysiological profile and has been consistently associated with a higher incidence of spontaneously emerging dreams (Hobson et al., 2000). In what way then, do these massive shifts in neuronal and cognitive activity associated with the different stages of sleep affect the dynamics of sleep patterns?

In this electrocorticography (ECoG) study, we addressed these questions by examining the spatial patterns of correlations of high-frequency (gamma) broadband power (HBB) across electrode pairs. We asked whether these correlation patterns observed during sleep (sleep patterns) may actually correspond to the spatial structure of functional patterns of sensory cortex re- 
Table 1. Patient information table

\begin{tabular}{lllll}
\hline Patient no. & Gender & Age & Electrodes & Location \\
\hline 1 & M & 28 & 49 & LH: Tem, Occ, Fro; RH: Tem, Fro \\
2 & M & 45 & 47 & LH: Tem Fro; RH: Tem, Fro \\
3 & F & 18 & 71 & RH: Tem, Par, Occ \\
4 & F & 41 & 41 & LH: Tem; RH: Tem, Par, Fro \\
5 & F & 33 & 53 & LH: Tem Fro; RH: Tem, Fro \\
6 & M & 36 & 66 & RH: Tem, Fro, Par, Occ \\
7 & F & 16 & 61 & LH: Tem, Fro, Par, Occ \\
\hline
\end{tabular}

Electrodes, Number of electrodes analyzed after subtraction of trigger electrodes (used to synchronize the stimuli and $E(\mathrm{O} G$ recordings) and electrodes with bad leads; $\mathrm{LH}$, left hemisphere; $\mathrm{RH}$, right hemisphere; Tem, temporal; $0 \mathrm{cc}$, occipital; Fro, frontal; Par, parietal; $M$, male; F, female.

sponses elicited by sensory stimulation during the awake state. Movie-viewing sessions were used to reveal the functional organization of these sensory patterns (movie patterns). Note that our study was aimed specifically at revealing the emergence of averaged spatial correlation patterns, which do not necessarily imply a "replay" (i.e., a repeat of unique temporal sequences of such activations; Staba et al., 2002; Ji and Wilson, 2007). We further examined to what extent these spatial patterns change from moment to moment, and particularly whether such dynamic changes may differ across different sleep stages.

Our results show that sensory cortex manifests spatially organized sleep patterns, which bear a significant similarity to the functional organization revealed during movie-viewing sessions in the awake state.

\section{Materials and Methods}

Data acquisition. Recordings of electrical activity were obtained from 388 recording sites in seven neurosurgical patients with pharmacologically intractable epilepsy (three male, four female), monitored for potential surgical treatment. Electrode location was based solely on clinical criteria. Electrodes covered most of the lateral and ventral surfaces of the cortex (for details, see Table 1). Each patient was implanted with subdural electrodes (Adtech), which were placed directly on the cortical surface. Each electrode was $2 \mathrm{~mm}$ in diameter, with $4-8 \mathrm{~mm}$ spacing between adjacent electrodes. Recordings were monopolar and were referenced to an extracranial electrode. The signal was sampled at a rate of 200 or $256 \mathrm{~Hz}$, and either filtered electronically between 1 and $70 \mathrm{~Hz}$ or high-pass filtered at $1 \mathrm{~Hz}$ (Grass Technologies). Stimulus-triggered electrical pulses were recorded along with the ECoG data (through trigger channels) to allow precise synchronization of the stimuli with the electrical responses. Channels with low signal-to-noise ratios were removed from the analysis.

All awake sessions were conducted at the patient's quiet bedside while the patient was sitting upright in bed, after periods of at least $3 \mathrm{~h}$ without any identifiable seizures. Stimuli were presented via a standard laptop screen.

Sleep data were collected from three seizure-free nights in which the patient slept for at least $6 \mathrm{~h}$. The first night was before movie exposure, while subsequent nights were after movie exposure. Patients provided written informed consent to participate in the experiment. The experimental protocol was approved by the Tel Aviv Sourasky Medical Center Committee for Activities Involving Human Subjects.

Electrode localization. Computed tomography scans following electrode implantation were coregistered to the preoperative MRI using iPlan Stereotaxy software (BRAINLAB) to determine electrode positions. The three-dimensional brain image thus mounted with electrode locations was normalized to Talairach coordinates (Talairach and Tournoux, 1988) and rendered in BrainVoyager (Brain Innovation) in two dimensions as a surface mesh, enabling precise localization of the electrodes both with relation to the patient's anatomical MRI scan and in standard coordinate space. For joint presentation of all patients' electrodes and to aid comparison with previous fMRI mapping performed in our laboratory, electrode locations were projected onto a cortical reconstruction of a specific healthy subject, which is routinely used to visualize results in our mapping studies.

Experimental design. Nine minutes of an audiovisual movie clip from the popular western movie The Good, the Bad, and the Ugly were presented twice to subjects, who were asked to watch and follow the plot (Fig. 1). A 30 min break, in which patients engaged in other tasks, separated the two presentations (for further details regarding this stimulus, see Mukamel et al., 2005).

Data during sleep were collected from the moment the video monitoring showed that patients were preparing for sleep until patients awoke in the morning. Sleep and sleep staging were confirmed later from the video monitoring and spectral analysis of the data (see below), and long periods of wakefulness ( $>8 \mathrm{~min}$ ) were removed from the data.

Data analysis. Data analysis was performed in MATLAB (MathWorks) using EEGLAB (Delorme and Makeig, 2004) and in-house code. As in our previous studies (Fisch et al., 2009; Privman et al., 2011), each electrode was dereferenced by subtracting the average signal of all other electrodes, thus discarding non-neuronal contributions. See the Sleep staging section below for details on how sleep staging was performed. For all other analyses, the signal was then band passed in the gamma (40-70 $\mathrm{Hz}$ ) frequency range using a linear-phase finite impulse response (FIR) filter, and the band-limited power (BLP) modulation extracted by taking the absolute value of the Hilbert transform (Lachaux et al., 2005). Forty hertz is the lower bound of the gamma frequency band, while our upper bound, $70 \mathrm{~Hz}$, was imposed by hardware constraints in the recording system. To remove the substantial high-frequency noise that was present in the recordings, a $1 \mathrm{~s}$ moving average temporal smoothing was applied to the signal. This resulted in the smoothed HBB of the signal, on which all further analysis (apart from sleep staging) is based. To compare HBB during stimulus presentations between electrode pairs, Pearson's linear correlation coefficient was computed separately for each pair of (smoothed, HBB) signals. All correlations analyzed were simple power correlations between the smoothed HBB signals of the different electrodes. Due to the substantial temporal smoothing, an examination of phase locking was not feasible for the present study.

Peaks in the data were removed by replacing outlying points $(>3 \mathrm{SD}$ above/below average) with the signal average. This procedure removed many of the remaining noise artifacts in the signal and minimized the effect of interictal events.

Movie correlations. The movie-induced pattern (i.e., the movie pattern) was revealed by mapping the matrix of correlations between each electrode and all other electrodes. Importantly, the correlation was calculated across movie presentations-meaning that the response of electrode $i$ during the first movie was correlated with the response of all other electrodes during the second movie and vice versa. Half of the correlation matrix was composed of the correlation of the HBB time courses of each electrode in the first movie presentation with the HBB time courses of all the other electrodes in the second presentation, while the second half is composed of the correlation of the HBB time courses of each electrode in the second movie presentation with the HBB time courses of all the other electrodes in the first presentation. This cross-presentation analysis ensured that correlations are not influenced by epileptic activity. The resulting mosaic could therefore be divided into two halves-below and above the diagonal. In the first half, below the diagonal, we plot the correlations of electrodes in the first movie presentation (along the $y$-axis) with electrodes in the second movie presentation (along the $x$ axis) and vice versa for the top half (above the diagonal). Note that these are two independent datasets. The diagonal is composed of the correlation of each electrode with itself across movie presentations. Sensory electrodes were defined as electrodes whose correlation with themselves across movie presentations exceeded the 95th percentile of random correlations (calculated from the distribution of sham correlations, see next section).

Sham correlations. To calculate the sham correlation structure (i.e., the sham pattern), the same procedure as above was followed, but for the second movie presentation the HBB time course of each electrode was shuffled by putting the second half of the time course in the beginning followed by the first half of the time course (for an illustration, see Fig. 1). In this manner, events in the two movie presentations were no longer 


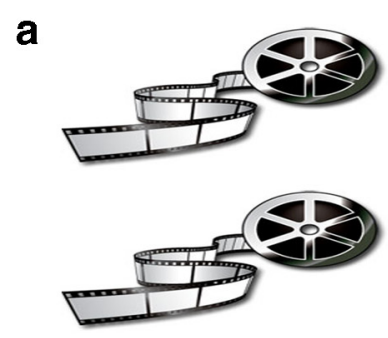

c Movie correlations

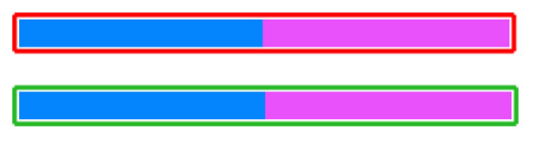

Run 1
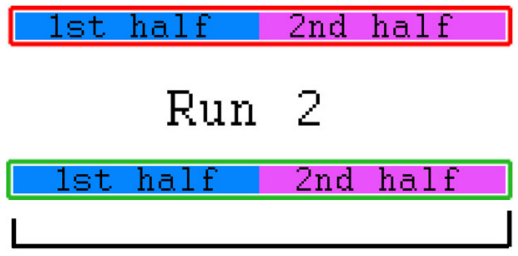

9 minutes

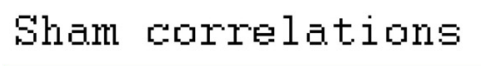

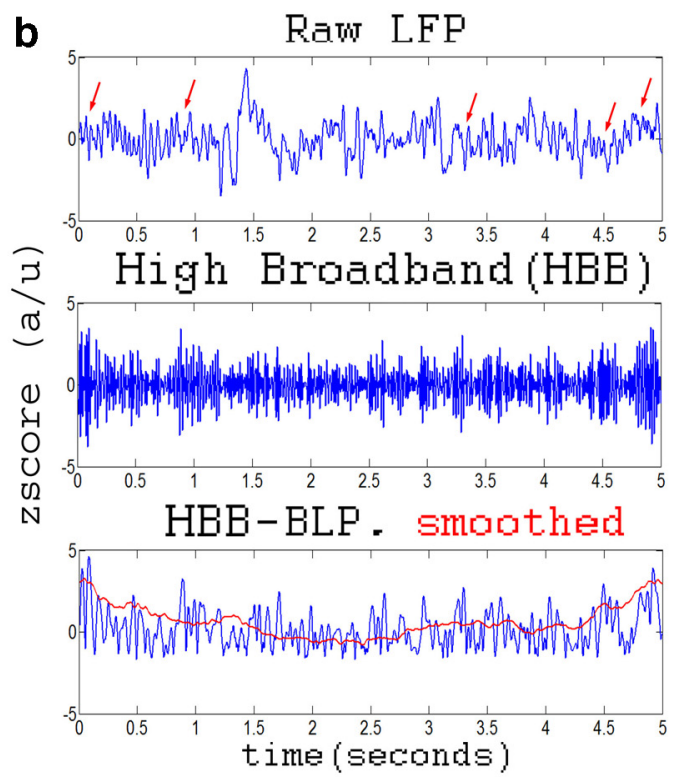

Figure 1. Experimental paradigm and calculation of HBB. $\boldsymbol{a}$, Experimental paradigm. A 9 min segment from the engaging film The Good the Bad and the Ugly was presented to patients twice. $\boldsymbol{b}$, Calculation of the smoothed HBB signal, which was used throughout the analysis. Arrows highlight times with high levels of gamma in the raw signal. c, Preparing the time courses for computing sham correlations: for each electrode in the second movie presentation, the time course was scrambled so that the second half of the time course was placed before the first half.

time locked or even overlapping, and though the basic spatial structure of the electrodes was maintained, no meaningful correlations were expected.

Sleep staging. The patients participating in this study did not have scalp electroencephalography (EEG) channels, or electro-oculography (EOG) or electromyography (EMG) recordings, making conventional scalp EEG-based sleep staging impossible, However, since all NREM sleep stages are characterized by an increased presence of waves in the delta frequency (slow waves in slow-wave sleep; K-complexes in stages 1-2), and since REM sleep is characterized by spectral content almost identical to wakefulness, we hypothesized that it would be possible to extract sleep stages, or at least the differentiation between NREM and REM/wakefulness, from analysis of the spectral content of the ECoG signal. Starting from the raw data, each electrode was dereferenced by subtracting the average signal of all other electrodes, thus discarding non-neuronal contributions. The signal was then band passed in the delta $(1-4 \mathrm{~Hz})$ frequency range using a linear-phase FIR filter, and the BLP modulation was extracted by taking the absolute value of the Hilbert transform. The delta BLP was then averaged across all electrodes and over $1 \mathrm{~min}$ (as sleep state changes are slow, global events). Moments with high delta power were taken to mean NREM sleep, whereas moments with low delta power were construed as REM sleep or wakefulness. To test this method, we calculated the same index for an independent set of sleep ECoG data, unrelated to our study, for which conventional staging was also available. This dataset was taken from patients implanted with microwires, so the signal there is even more localized than the local field potential (LFP) available from our patients. The results of this analysis are shown in Figure 7, and the remarkable fit is clearly evident.

Our measure of delta was not sufficient for discerning between REM sleep and wakefulness, for which the EOG and EMG channels are particularly useful in conventional staging. Instead, we used the video recordings to determine whether patients were awake or asleep, and supplemented this with the prediction from normal sleep cycles. For the sake of prudence, analyses relying on the differentiation into REM/NREM stages, such as the relationship of the staging to the sleep patterns, used only parts of the night clearly identified beyond doubt as NREM/REM sleep (delta power $>1.5$ SD from the average for NREM, delta power $>1.5$ SD less than average for REM, and no signs of wakefulness in the video for at least 3 min prior to and after). Our algorithm correctly identified $100 \%$ of the REM/NREM intervals that met these criteria for the dataset for which independent, conventional scalp-based sleep staging was also available (see Fig. 7). Apart from the independent dataset shown in Figure 7, all other sleep staging shown (see Fig. 8) or used in this article is based on the above algorithm.

Sleep. Sleep data were preprocessed in the same manner as the movie data. For each patient and each night, the data were divided into $5 \mathrm{~min}$ time windows, with a sliding 1 min window (so that a 4 min overlap exists between two adjacent windows). For each $5 \mathrm{~min}$ time window, the matrix of correlations of HBB modulations between each electrode and all other electrodes was calculated ("momentary correlations") in a similar fashion to that described for the movie-induced correlation. However, importantly, during sleep the correlations were computed during the same time segment rather than across different presentations, as was done for the repeated movie stimuli. As with the movie data, peaks in the data were removed by replacing outlying points ( $>3 \mathrm{SD}$ above/below average) with the signal average, removing the remaining noise artifacts in the signal and minimizing the effect of interictal events. To create an overall average correlation matrix for each patient, the correlation matrices from all non-overlapping time windows were averaged across all 3 nights of each patient. Apart from the general average sleep matrix, a stage-specific REM/NREM template was created for each subject by averaging all the momentary correlation matrices belonging to that sleep stage according to the stricter criteria mentioned above.

Correlations between the sleep and movie-driven patterns (i.e., endoactivation), and between sleep and sham patterns (i.e., sham correlations), as well as those between the momentary correlations and the stage-specific sleep templates and the odd/even time windows during sleep in general, were quantified by calculating the Pearson's linear correlation coefficient between matrices (after transforming them to vector form). For sleep, only half of the data in each matrix were used (because of the inherent symmetry). For the movie/sham structures, correlations were first averaged across the diagonal, between the two independent datasets (see explanation in the Movie correlations section). The dissimilarity of each momentary correlation to its relevant template (see Fig. 9, distance from template) was defined as 1 - the correlation (Pearson's correlation coefficient) between the two. In addition to these measures, we also calculated the "global" or averaged correlations. This was computed by simply averaging all correlation values across all electrode pairs and across all the momentary correlation matrices.

Visualizing endo-activation. To visualize the emergence of the moviedriven patterns during sleep (Fig. 4), the following procedure was used. First, all electrode pairs were rank ordered according to their sensory- 
driven correlation during the movie-viewing task (i.e., the correlation of each electrode pair across movie presentations). Next, we arranged the electrode pairs in an outward spiral, starting from the middle of the square (highest correlation) to create an easily recognizable pattern, with highly correlated electrode pairs in the center, and correlations decreasing away from the center. Note that anatomical considerations played no part in the creation of this pattern, which was a simple, artificially constructed pattern designed to highlight in a convenient manner the reemergence of the same pattern during sleep. The color code in left panels of Figure 4 indicates the correlation value for each electrode pair across movie presentations. For the analysis of sleep patterns (Fig. 4, middle), the order of electrode pairs was maintained. However, the color coding now reflected the correlation values of these electrode pairs during sleep (Fig. 4, middle panels). If indeed the spontaneously emerging sleep pattern was similar to the movie-driven pattern, we would expect to see a correlation matrix revealed during sleep similar to that seen during the movie.

For the right panels of Figure 4, the color code again reflects the correlation values of the electrode pairs during sleep. However, electrodes were rank ordered and arranged in the panel not according to their movie-driven correlation level, but rather according to their correlation values in the sham condition.

Permutation tests. Permutation tests were used to obtain significance measures for most of our analyses. This nonparametric method was chosen so that no additional assumptions needed to be made on the data (such as normal distribution). This method preserves the inherent properties of the data and corrects for multiple comparisons. For the permutation test used in Figure 5, the electrode labels of the movie correlation structure were shuffled, meaning that overall the values in the movie pattern remained the same, but their order within the pattern was changed randomly. This procedure was repeated over 10,000 iterations with replacement, and the endo-activation was calculated between the sleep correlation structure and the shuffled movie correlation structure.

For the permutation test of the significance of the correlation to staging shown in Figure 8, we first calculated separately the average endoactivation during all time windows in each night for each patient defined as REM/NREM sleep, and calculated the difference between the average NREM/REM endo-activations. The REM/NREM labels were then shuffled over 10,000 iterations with replacement (meaning time windows were randomly assigned as belonging to either REM or NREM sleep), and the difference in endo-activation between NREM and REM shuffled data was calculated for each iteration. A similar method of shuffling the REM/NREM labels across 10,000 iterations was used to calculate the permutation tests for the variance in correlation with the sleep template, the increase in correlation between the template and the movie versus the momentary correlations, and the difference between the REM and NREM sleep templates. Note that all these tests are based on measuring the average differences between only two conditions (REM vs NREM sleep, template vs average momentary correlations). This comparison is therefore a single measurement that does not require a multiplecomparison correction.

Endo-activation contribution. To calculate the contribution of each electrode to the endo-activation, we calculated the difference between the endo-activation of the full structure and the structure without that electrode (i.e., all the correlation values of that electrode with those of all other electrodes). The sensory sensitivity index plotted in Figure $5 b$ (inset) was defined as the correlation of that electrode with itself, across the two movie presentations.

Fine-structure analysis. Testing whether the endo-activation could be the result of broad changes in connectivity caused by global, nonspecific waves across large areas of cortex was performed by selecting for the analysis only the electrodes that were scored in the top $15 \%$ of contributions to endo-activation. Next, we removed (projected out) the global component (i.e., the average HBB signal across all these electrodes) from the response of each electrode. This procedure effectively removes any large-scale fluctuations from the data. We then recalculated the endoactivation in the absence of such global correlated activations.

Neighbor removal. For each patient, the average Cartesian anatomical distance between adjacent electrodes on a strip or grid was calculated for

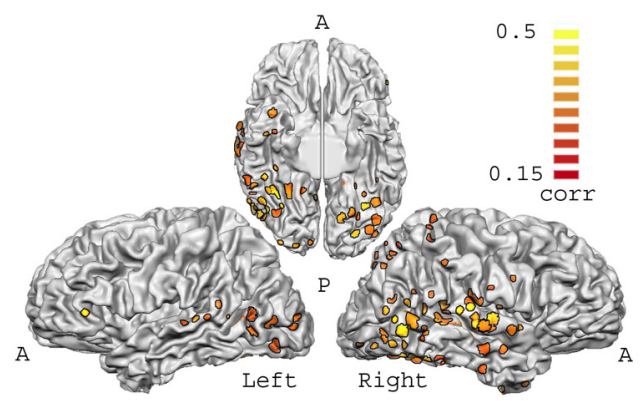

Figure 2. Location of sensory electrodes. Location of all sensory electrodes (electrodes whose HBB time course was highly reproducible across movie presentations) of all subjects on the brain. Electrodes are color coded according to their cross-movie correlation. A, Anterior; $P$, posterior.

all electrode pairs. Neighbors were defined as electrodes closer than 1.4 times this average distance. We then calculated for each patient the distance between each electrode pair, and defined a subset of the correlation matrix that excluded neighboring electrodes. To remove the secondnearest neighbors, neighbors were defined as electrodes closer than 2.5 times the average distance between adjacent electrodes.

\section{Results}

Our study was based on 388 intracranial recordings sites obtained from seven patients (see Table 1). During the waking state, patients were presented with two repeats of the same 9 min segment of a movie (Fig. 1a), and the patients' task was to passively view the movie. The movies were presented with a minimal separation of $30 \mathrm{~min}$. Following previous work showing a high degree of correspondence between modulations in HBB and spiking (Nir et al., 2007; Fisch et al., 2009; Kang et al., 2010; Mukamel et al., 2011), our analysis focused on changes in power in the $40-70 \mathrm{~Hz}$ frequency range (Materials and Methods; Fig. $1 b$ ). While other frequency bands may be informative as well (Sederberg et al., 2003; Mukamel et al., 2005; Canolty and Knight, 2010; Donner and Siegel, 2011), it should be noted that these lower frequencies are far less localized in space (Privman et al., 2013) and are therefore not suitable for the analyses performed here, which rely on the highly localized nature of the high-frequency signals (for details, see Discussion). HBB was strongly modulated during the movie presentations. Our analysis focused on correlations between electrodes across the two movie presentations, indicating activity that was time locked to events in the movie. As in previous research from our laboratory with these movies, we found that in a subset of electrodes these HBB time courses were significantly reproducible across movie repeats, indicating that they were driven by the naturalistic audio-visual information (see also Hasson et al., 2004). Such cross-presentation correlations could only be generated by events in the movie to which the electrode responses were time locked in a reproducible manner. Electrode activity associated with spontaneous/ongoing or epileptic interictal noise should not show such time locking to the sensory stimulation. Thus, analysis of such cross-presentation correlations allowed us to separate the contribution of purely sensory-/ movie-driven responses from spontaneous noise and interictal activity. The electrodes that showed the most significant $(p<$ $10^{-4}$, permutation test) cross-presentation correlations are depicted in Figure 2.

The movie-driven cross-presentation correlations could be found also across pairs of electrodes (Fig. $3 a$; Materials and Methods), demonstrating that the movie reliably activated cortical networks in a correlated manner and not only isolated sites. 


\section{Movie}

correlations

a
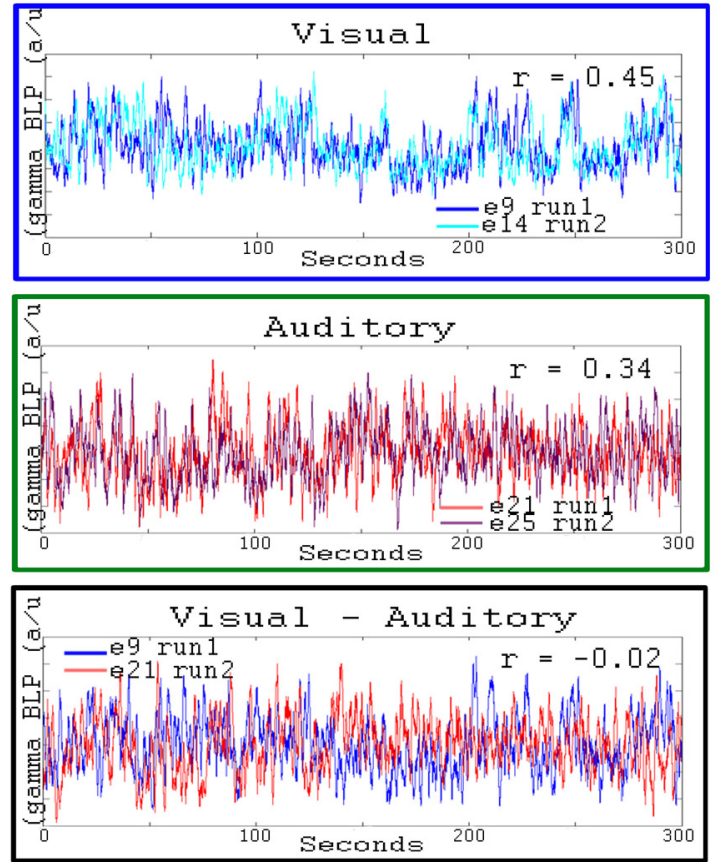

b

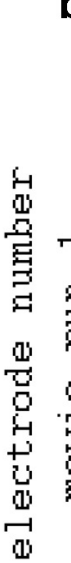

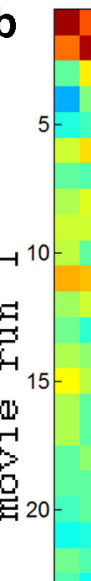
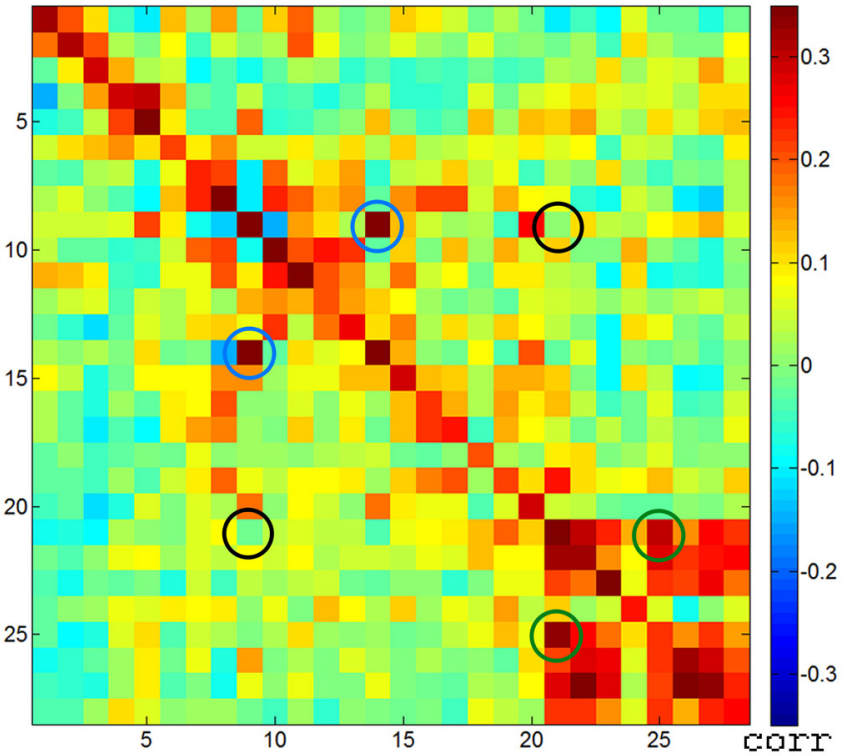

electrode number movie run 2

\section{Sleep}

correlations
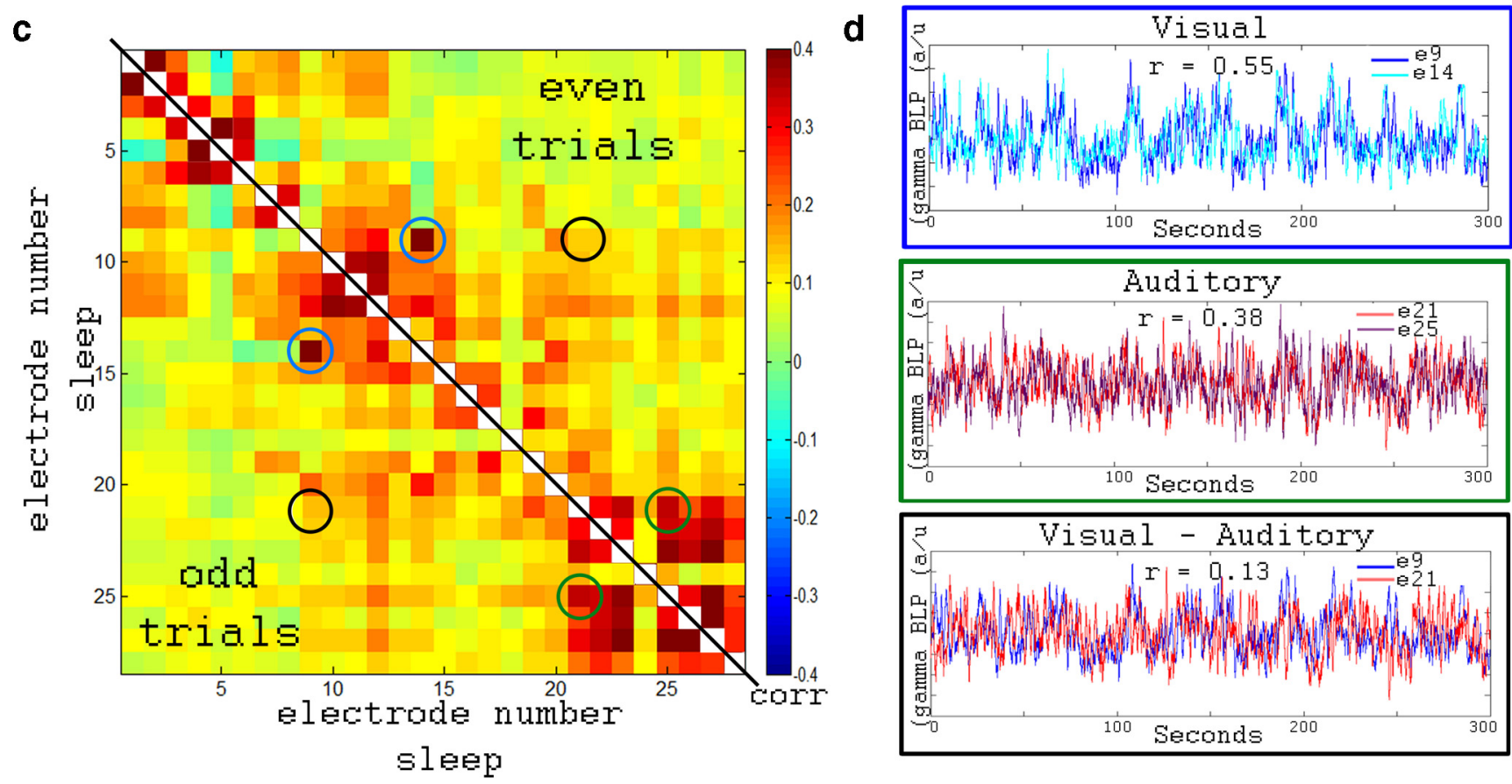

Figure 3. Example of functional organization patterns during awake and sleep states. $\boldsymbol{a}$, Example time courses from a single patient from four different visual and auditory electrodes. The time courses of one visual and one auditory electrode were taken from the first movie presentation, and the others from the second movie presentation. Note the strong within-category correlation across movie repeats and the lack of between-category correlation. $\boldsymbol{b}$, Correlation structure of all the sensory electrodes reflecting the underlying functional organization. The bottom lefthalf depicts the correlation between the time course of each electrode in the first movie presentation (along the $y$-axis) and the time courses of electrodes in the second movie presentation (along the $x$-axis), while the top right half depicts the inverse. The diagonal depicts the correlation of each electrode with itselfacross movie presentations. Note the remarkable reproducibility of the functional organization reflected in the mirror symmetry across the diagonal, despitethese being two independent datasets. The visual electrode pair plotted in $\boldsymbol{a}$ is circled in blue, the auditory electrode pair is circled in green, and the visual-auditory pair is circled in black. Inset, The location of these four electrodes on the brain. $c$, Average correlation structure of all sensory electrodes for all odd (bottom left) and even (top right) time windows during sleep. Same presentation and electrode pairs circled as those in $\boldsymbol{b}$. This time the two diagonal halves separated by the black line are a combination of two separate matrices, depicting odd and even time windows — note again the highly reproducible symmetrical pattern, but also the robust common activation element (reflected in overall brighter colors) in the sleep pattern. $\boldsymbol{d}$, Time courses of the same four electrodes as in $\boldsymbol{a}$ during 5 min of sleep. e, Electrode. 
Importantly, these movie-induced correlations were not uniform across cortical recording sites, as would be expected from a globally fluctuating network, but rather showed a fine-grained, functionally organized mosaic (Fig. 3b). The spatial pattern of correlation (e.g., the identity of highly correlated or anticorrelated pairs) was highly consistent, as illustrated by the symmetry of the correlation matrix, in which the two halves of the patterns were derived from two independent measures of the correlation matrix: the bottom left half of Figure $3 b$ depicts the correlation of the time courses of each electrode in the first movie presentation with the time courses of all the other electrodes in the second presentation. The top right half of Figure $3 b$ depicts the complementary independent set-i.e., the correlation of the time courses of each electrode in the second movie presentation with the time courses of all the other electrodes in the first presentation (see Materials and Methods). The diagonal in Figure $3 b$ shows the correlation of each electrode with itself across movie presentations. As can be seen, the two halves are highly symmetric, reflecting the consistent nature of the movie-driven functional organization underlying these correlations.

During sleep, HBB again showed robust fluctuations (Fig. 3d). Furthermore, specific electrode pairs showed a high degree of correlated fluctuations (Fig. $3 c, d$ ).

To examine whether this sleep pattern was similar to the movie-driven patterns obtained when subjects were awake, our analysis proceeded in two stages. We first analyzed the entire sleep period, and then separately examined the sleep patterns during REM and NREM sleep stages. Examining the averaged sleep pattern of electrode correlations, regardless of staging (Fig. $3 c$; Materials and Methods), showed clear and consistent sleep patterns that were not uniform (albeit during sleep there was a greater component of global, i.e., average, correlation, as has been shown previously; Nir et al., 2008).

To demonstrate that the average sleep pattern was consistent and not a mere product of averaged noise, we divided the dataset during sleep into odd and even non-overlapping 5 min time segments, and averaged each set of segments separately to create two independent sleep templates (i.e., correlation matrices). Our results show that, indeed, the sleep patterns were highly consistent within individual patients. To visualize the striking reproducibility of the sleep patterns we plotted the odd and even trials in the same matrix. This is illustrated in an example depicted in Figure $3 c$, whereby the bottom left half of the pattern is derived from the averaged odd windows, and the top right pattern is derived from the (independent) even windows. Note that the two patterns show nearly perfect symmetry $(r=0.99)$, indicating a highly reproducible pattern during odd and even runs.

Next, we examined whether these consistent sleep patterns (Fig. $3 b$ ) showed any similarity to the sensory patterns revealed by the movie stimulation during the awake state (Fig.

\section{Patient 3}

Sleep correlations sorted by movie

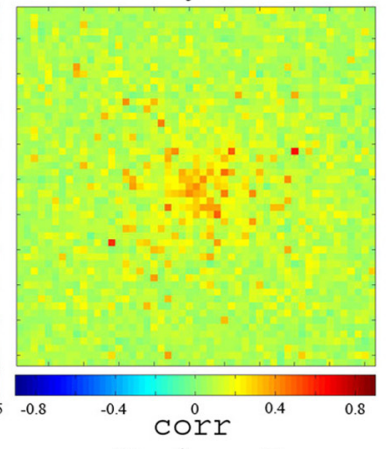

Patient 7

Sleep correlations sorted by movie

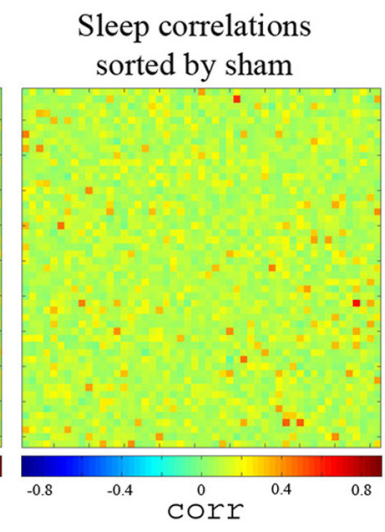

Sleep correlations sorted by sham

\section{Sleep correlations sorted by sham}
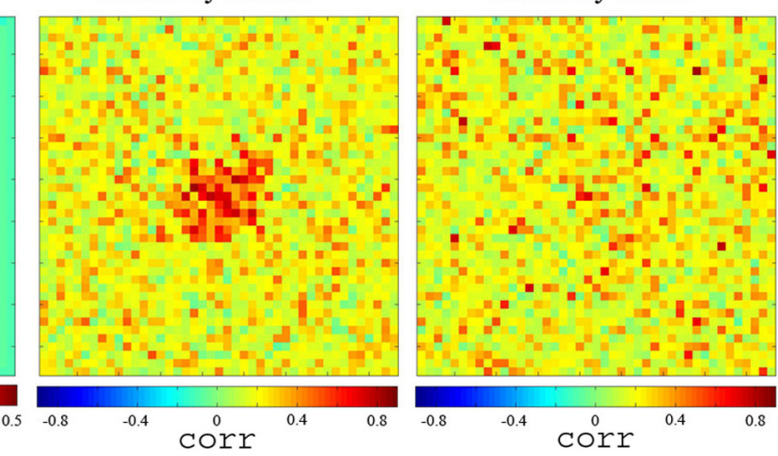

\section{.}

Figure 4. Visualizing the similarity of sleep patterns to sensory functional organization. Examples of endo-activations in two in of sleep, only this time electrode pairs were ordered according to the sham correlations (see Materials and Methods). Note the lack of any structure in sleep when ordered according to the sham correlations. corr, Correlations.

$3 b)$. Quantitative examination of the sleep patterns revealed that they were indeed significantly similar to the patterns revealed by the movie stimulation. Specifically, a highly significant ( $p<10^{-4}$, permutation test; Materials and Methods) correspondence was found between the cross-movie correlation matrix during the awake state (movie pattern) and the average correlation pattern emerging spontaneously during sleep (sleep pattern). This effect was significant in each patient individually and in the pooled data of all patients. In the rest of the manuscript, we will refer to the emergence of such similarity between the sleep patterns and the sensory functional organization (movie pattern) as "endo-activation."

Because of the complexity of the movie and sleep patterns, it was difficult to quickly discern by visual inspection to what extent they were similar to each other. To assist in such visual demonstration of the endo-activation phenomenon, we rank ordered all electrode pairs according to their level of sensory-driven correlation during the movie-viewing task and arranged them in an outward spiral, so that the electrode pair with highest correlation was in the center. Two examples of this procedure are depicted in Figure 4 (left panels; for details, see Materials and Methods). Keeping the same organization of electrode pairs, we then color coded each point by the level of its correlation during sleep. If the electrode pairs recaptured the same correlation structure during 


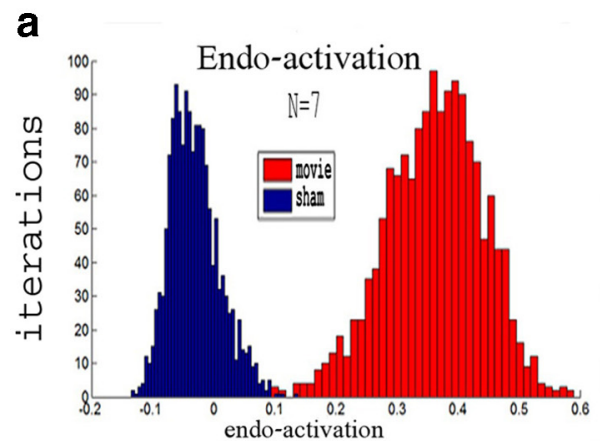

b

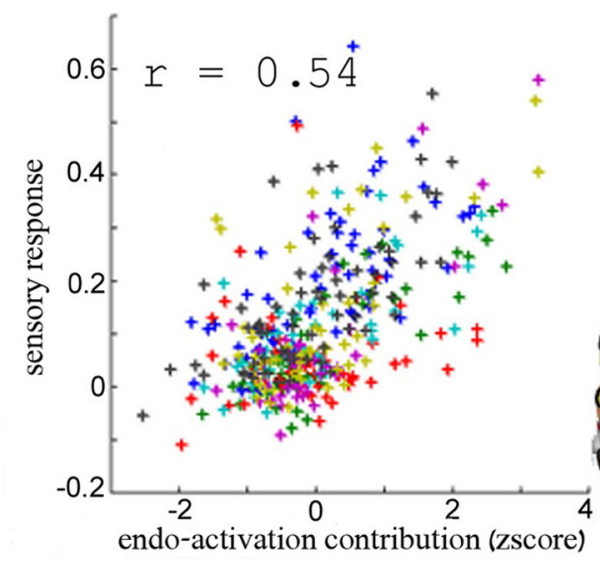

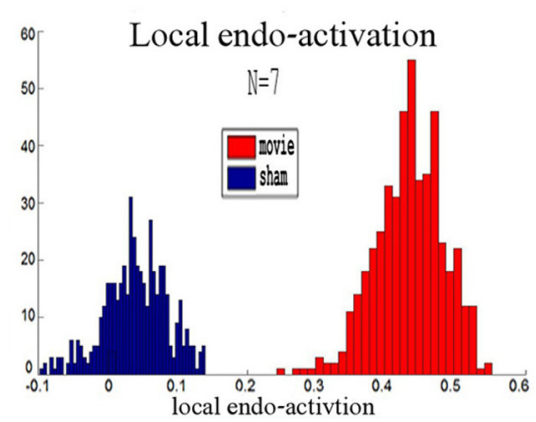

Left

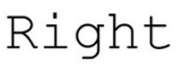

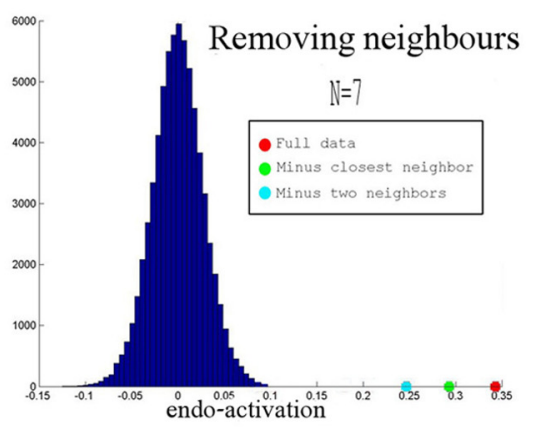

A
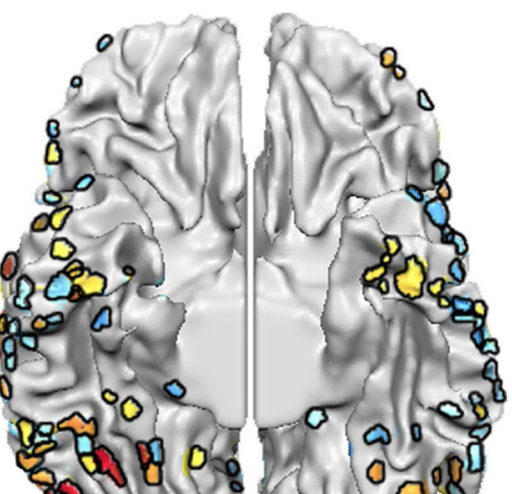

high positive contribution

(arbitrary units)

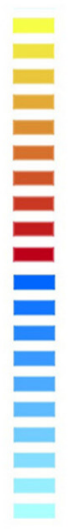

high negative contribtion
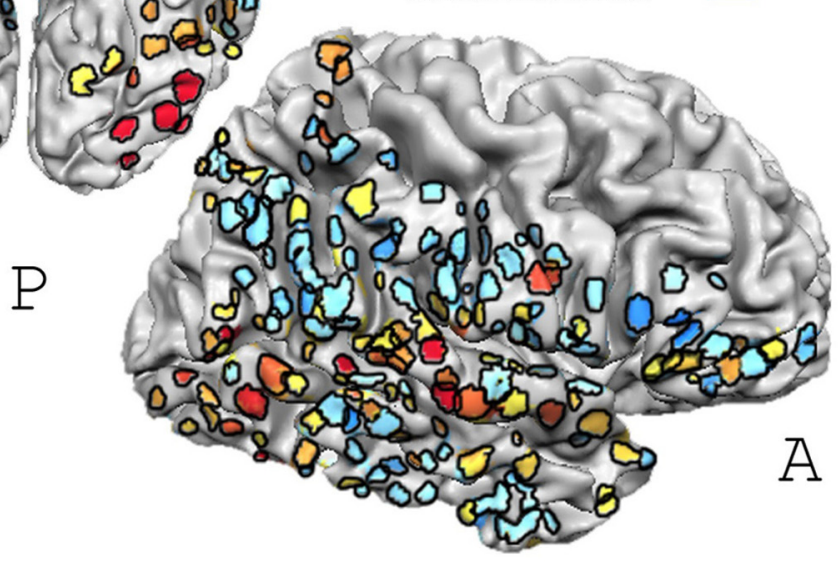

Figure 5. The local component of endo-activation patterns. $\boldsymbol{a}$, Left, Distribution of real endo-activation and sham levels across all time windows during sleep [correlation of the pattern in all sleep time windows with the cross-presentation movie pattern (red) and the sham pattern (blue)]. Middle, Distribution of endo-activation and sham of only the electrodes that contributed most to endo-activation, after projecting out their common signal. Right, The distribution (blue) of the endo-activation permutation test (see Materials and Methods). Red, green, and cyan dots indicate the means of the distributions of full endo-activation (depicted in the histogram in the left panel), endo-activation without a nearest neighbor, and endo-activation without two nearest neighbors, respectively. $\boldsymbol{b}$, Location of all the electrodes of all subjects on the brain, color coded according to their contribution to endo-activation. Note the clustering of highly contributing electrodes in sensory cortices. Inset, The relationship between endo-activation contribution and sensory sensitivity; colors in the inset reflect data from different subjects. A, Anterior; P, posterior.

sleep as they did during the movie stimulation, we would expect the same pattern generated in Figure 4 in the left panels to reemerge in the middle panels As can be seen in these examples, the pattern that emerged during sleep (Fig. 4, middle panels) was indeed visibly similar to that generated by the movie (Fig. 4, left panels), indicating a significant endo-activation of the original pattern during sleep. In contrast, rank ordering the electrode pairs not according to the actual movies, but according to sham correlations generated by correlating the movie with a shuffled version of it (Fig. 1c; Materials and Methods), failed to reveal any structure (Fig. 4, right panels; Materials and Methods). Note that this visualization does not contain information regarding the anatomy of the correlations, but is simply a means of having endo-activation, should it exist, "pop out."
Could the endo-activation be the result of the specific sensory experience generated by the movie before falling asleep? Comparing the endo-activation during sleep periods that occurred after movie exposure to those before movie exposure did not reveal a significant difference ( $p=0.27$, paired $t$ test), indicating that the observed endo-activation was not a direct replay of the previous movie stimulation.

Examination of the anatomical distribution of electrodes contributing to the endo-activation for the combined data from all subjects, and for each subject separately (Materials and Methods; Figs. $5 b, 6)$, revealed that they were clustered in sensory cortex, and indeed there was a significant correlation between sensory sensitivity, defined by the cross-movie presentation correlation of each electrode with itself, and endo-activation contribution 

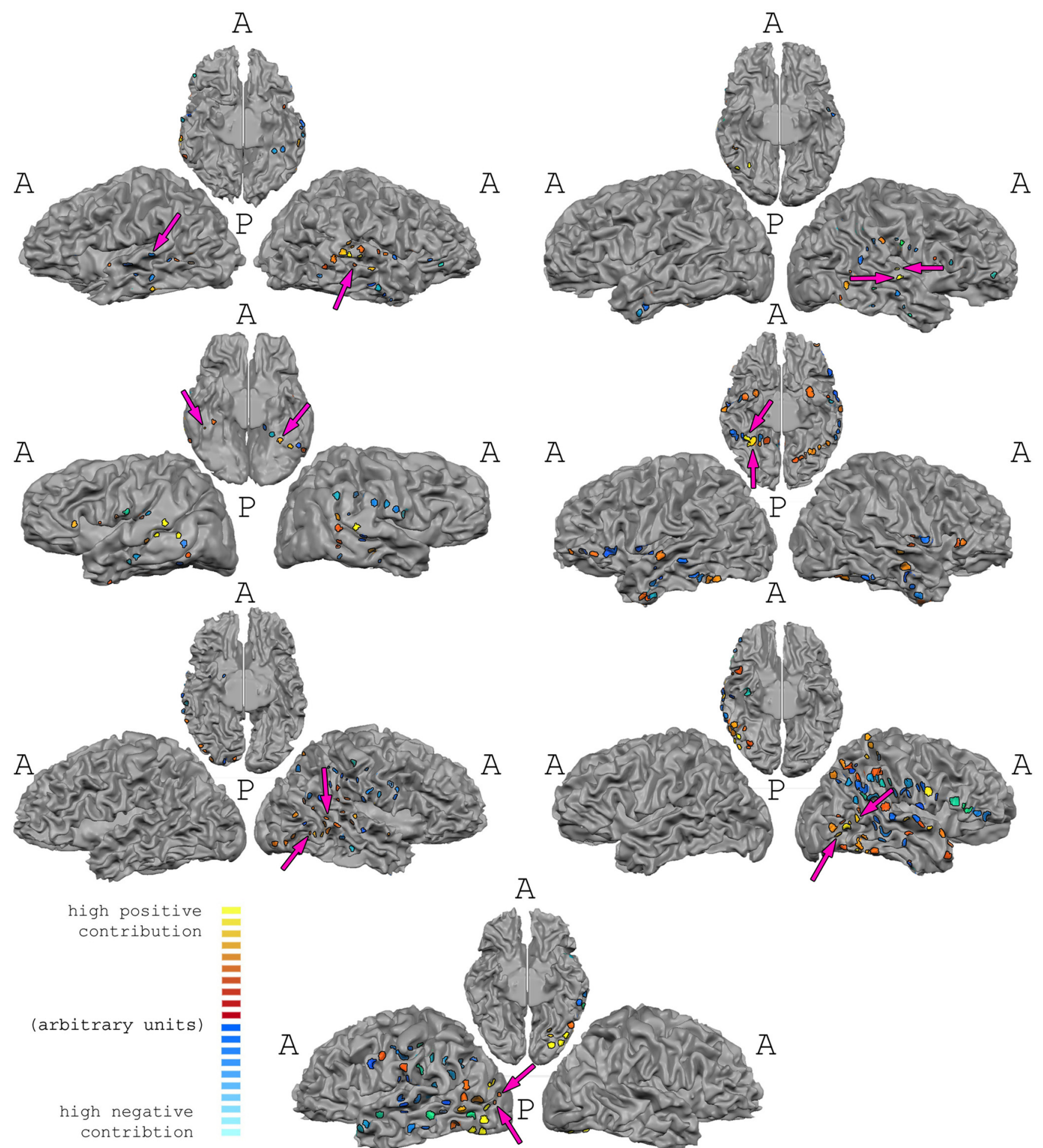

\section{A}
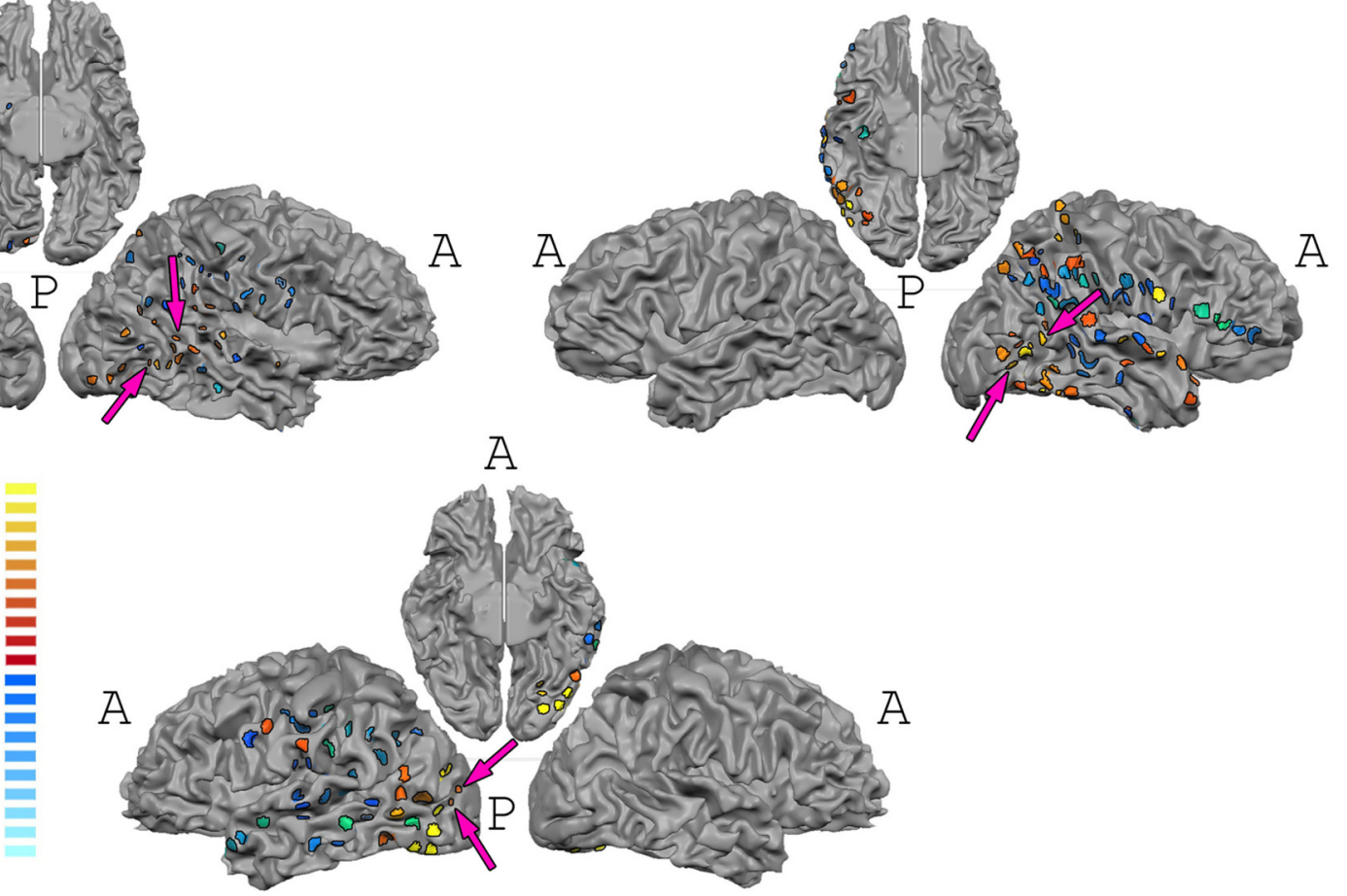

Figure 6. Endo-activation per patient. Electrode locations for each patient, shown on a reconstruction of his or her own brain. Electrodes are color-coded according to their contribution to endo-activation (compare Fig. $5 b$ ). Arrows show the pair of electrodes most highly correlated across movie presentations. A, Anterior; P, posterior.

(permutation test, $r=0.54$; Fig. $5 b$, inset; for details of analysis, see Materials and Methods).

Was the endo-activation driven by a uniform global fluctuation in sensory cortex in which all electrodes fluctuated in a correlated fashion, or was it the result of finer "microstructures within sensory areas, as hinted by the heterogeneous mosaic of the correlation matrices? To examine this point in more detail, we calculated the correlation pattern separately for each $5 \mathrm{~min}$ seg- ment of the time course throughout the night (see Materials and Methods). For each $5 \mathrm{~min}$ segment, we then recalculated the endo-activation level after removing (projecting out) the global signal (i.e., signal fluctuations that were common to all sensory electrodes; see Materials and Methods). Comparison to the sham data and a permutation test validated that the endo-activation, even after global network activations were removed, was still well outside the random distribution $\left(p<10^{-4}\right)$, indicating that the 


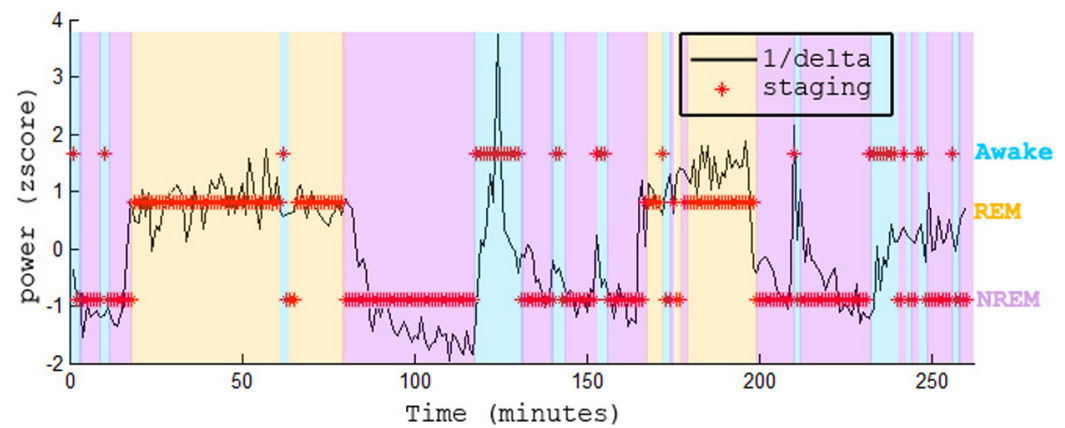

Figure 7. Sleep staging from intracranial recordings. The inverse of the average delta power calculated from the LFP of all leads in this dataset throughout the night (black trace). Colors and stars indicate the sleep staging derived in a conventional manner (surface EEG, EOG, and EMG; see Materials and Methods) by a sleep expert. Blue denotes episodes scored as awake, orange denotes periods scored as REM sleep, and purple denotes periods scored as NREM sleep. Note the remarkable fit between the two, with the delta power clearly distinguishing between REM sleep/wakefulness and NREM sleep. Video monitoring was used to distinguish REM sleep from wakefulness.

endo-activation was not driven solely by uniform signal fluctuations (Fig. $5 a$, middle).

Another potential source for the endo-activation could be spurious correlations due to direct current spread across neighboring electrodes. To rule out the possibility that the endo-activation was driven by neighborhood correlations, the endo-activations of all time segments were recalculated on a subset of the correlation matrices, from which the nearest neighbors were removed (see Materials and Methods). The endo-activation of these subnetworks, which contained no immediately adjacent electrodes, was still entirely outside the random distribution (sham correlations, also permutation test, $p<10^{-4}$; Fig. $5 a$, right). As a further test, the correlation patterns were recalculated, this time removing two nearby electrodes (so that the minimal interelectrode distance was $30 \mathrm{~mm}$ ). Again, the results showed a highly significant endo-activation (permutation test, $\left.p<10^{-4}\right)$.

So far we have examined the "static" aspects of the endoactivation (i.e., the correlation pattern averaged across the entire night). However, it is of interest to examine the possible dynamic aspects of such patterns (i.e., whether these patterns changed from moment to moment as sleep progressed). To reveal these dynamic aspects, we used the 5 min segments of the signals recorded during the night (as explained above). Examining the correlation patterns derived from these segments uncovered a high degree of moment-by-moment fluctuations.

We first compared the distribution of endo-activation during sleep across all $5 \mathrm{~min}$ segments to our sham control (for each time window: the correlation of the movie structure and the sleep correlation structure vs the correlation between the sham correlation structure and the sleep correlation structure). This last analysis is shown in Figure $5 a$ (left), and, as can be seen, there was virtually no overlap between the real and the sham distributions (for details, see Materials and Methods). However, there was a very wide range of endo-activation values, indicating that there were indeed substantial dynamic changes in the sleep patterns throughout the night.

We next turned to study whether these dynamic fluctuations in endo-activation were related to the sleep stages of the patients. To examine this aspect, we quantitatively defined a "staging index" based on the well established changes in the spectral contents of field potential (i.e., the massive increase seen in delta power in NREM vs REM sleep and wakefulness; Materials and Methods; Fig. 7). Note that this method cannot be used to distin- guish between REM sleep and wakefulness, for which purpose the video monitoring of the patient was used.

The first hint of a difference in the nature of dynamic fluctuations of patterns between the different sleep stages was found in the similarity of the sleep patterns to the movie-driven patterns (endoactivation). Thus, we found a stronger tendency for the moment-by-moment sleep patterns to resemble the moviedriven patterns during NREM compared with REM sleep stages, as is illustrated in Figure 8 (bottom). This was true for each subject individually, in itself resulting in a nonparametric significance level of 0.016 $\left(1 / 2^{6}\right)$. This observation was further verified using a permutation test on the segmented REM and NREM sleep stages (for details, see Materials and Methods). This analysis revealed that NREM sleep manifested significantly higher levels of endoactivation compared with REM sleep stages $\left(p<10^{-3}\right.$, paired $t$ test; Fig. 8, permutation test).

What could be the source of the higher endo-activation level during NREM sleep? One possibility could be a change in the range of different momentary patterns that appeared in the different sleep stages. To examine this possibility, we compared, in each state, all of the momentary correlation patterns (calculated separately for each $5 \mathrm{~min}$ of sleep) to a template of the average correlation pattern of that sleep stage. The template was calculated by averaging all the momentary patterns of that sleep stage (see Materials and Methods). Momentary correlations during REM sleep were significantly more variable and less similar to the REM template than were NREM sleep momentary correlations to the NREM template, indicating a substantial change in the behavior of functional organization between the two states $\left(p<10^{-4}\right.$, permutation test; the average distance from template during REM sleep, 0.31; average NREM sleep correlation to template, 0.15 , see Materials and Methods).

Could the increase in pattern variability between REM and NREM sleep be due simply to individual electrodes becoming noisier during REM sleep compared with NREM sleep? It should be noted that increased noise added independently to each electrode should disrupt the global (averaged) correlation strength between all electrode pairs. If REM sleep was indeed characterized by greater local noise, then we would expect a decrease in the global level of correlations during REM. However, we found no consistent difference between these global correlations when comparing REM and NREM sleep stages across subjects.

We next examined whether changes in the global correlation (reflecting changes in local noise) may explain the observed changes in pattern variability during the different stages. The results are displayed in Figure 9, which plots the amplitude of pattern variability [i.e., the dissimilarity (distance) of each momentary pattern and the averaged stage-specific template pattern, or "distance from template"] against the global correlation for each 5 min segment. Apart from a minority of outliers $(<5 \%$ of the data) mostly caused by global noise, the bulk of global correlations fell within a narrow window, and within this window there was no relationship between the global (average) correlation and the pattern distance from the mean stage-specific template. This result argues against local noise as the source of the increased pattern variability during REM. The individual mo- 

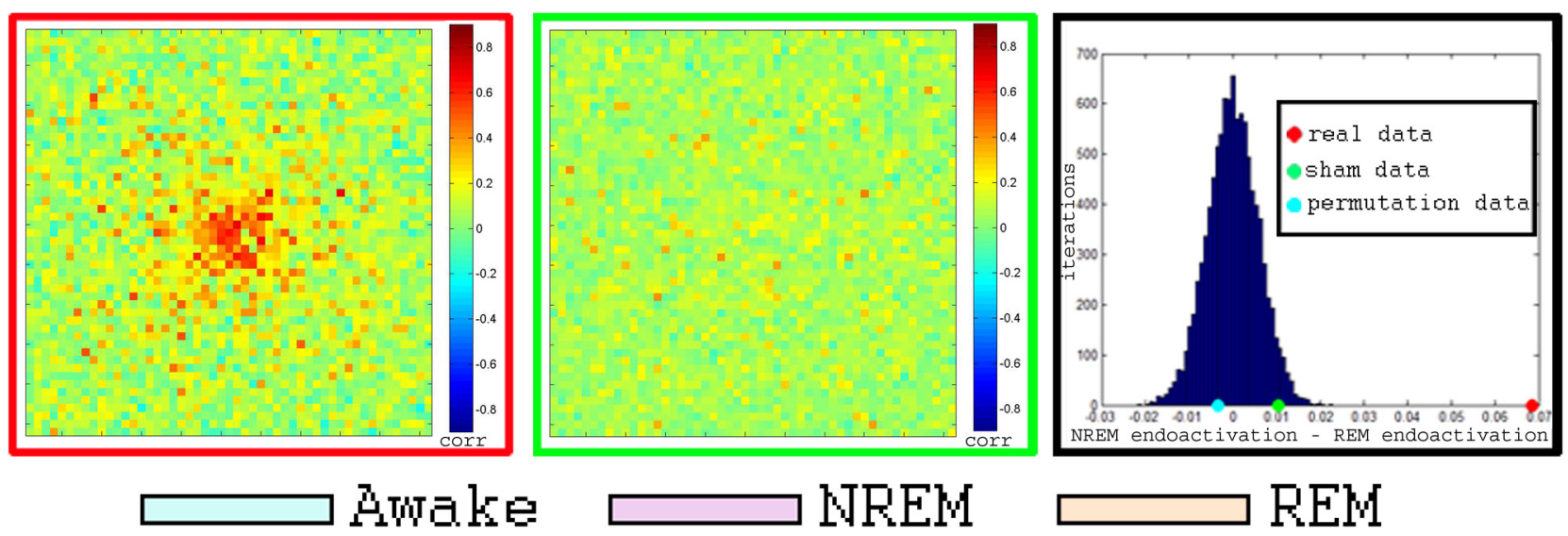

\section{REM}

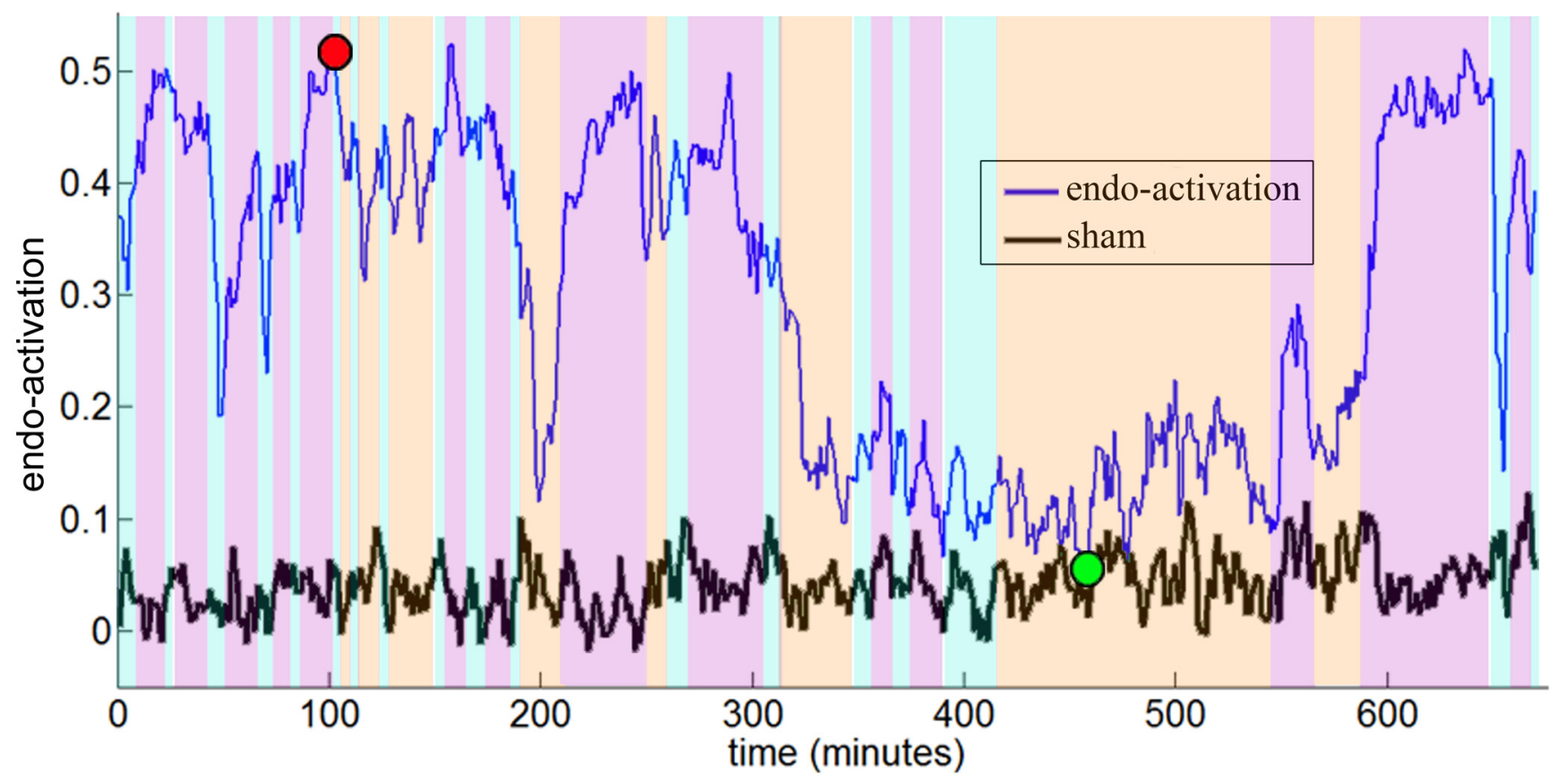

Figure 8. Endo-activation dynamics. Degree of endo-activation throughout the night, superimposed on the various sleep stages. Note the consistently higher endo-activation during NREM sleep. Using the same visualization as in Figure 4, the red frame illustrates electrode correlations during sleep taken from a moment with high endo-activation (marked by red dot), while green shows the same electrode correlations taken from a moment of low endo-activation (marked by green dot). The black frame shows the average difference between NREM and REM endo-activation for all subjects (red dot) versus a random distribution (permutation test: blue, distribution; cyan, mean), and the mean NREM-REM difference for sham data (green).

mentary REM sleep patterns were more distant from the average REM template than NREM sleep patterns (Fig. 9), indicating that in general the NREM patterns were more stable, whereas during REM sleep a larger range of patterns was activated at different times.

Although the REM and NREM templates were very similar ( $r=0.87, \mathrm{SD}=0.014$, averaged across subjects), there was still a significant difference between them, as revealed by a permutation test ( $p<10^{-4}$, see Materials and Methods).

Could the increased endo-activation during NREM sleep and the decreased range of momentary patterns be due either to higher levels of HBB power (i.e., a higher signal during NREM sleep) or, alternately, to higher amplitude of signal fluctuations, again leading to a higher signal-to-noise ratio? To examine these possibilities, we checked the difference between average HBB during NREM and REM sleep, and found an opposite effect. HBB was significantly reduced in NREM compared with REM sleep $\left(p<10^{-16}\right.$, paired two-tailed $t$ test). The amplitude (power) of HBB fluctuations was similarly reduced ( $p<10^{-18}$, paired two- tailed $t$ test) in REM sleep. Such a reduction in high-frequency broadband power and variance could not account for the observed increased endo-activation during NREM sleep.

\section{Discussion}

Our study reveals that spatial patterns of coherently fluctuating ECoG signals generated during sleep (sleep patterns) are not random but show a highly significant similarity (called here endoactivation) to the functional organization (i.e., correlation) patterns that emerge during naturalistic audio-visual stimulation (a segment of an engaging movie) in the awake state (Figs. 5, 6). In other words, the functional organization patterns that are revealed through sensory stimulation in the awake state emerge spontaneously during sleep.

As expected from our previous studies using movie stimulation (Hasson et al., 2004), the sensory-responding electrodes contributing to these patterns were located in visual and auditory cortices, as well as some motor and frontal areas (Figs. 2, 5b). 


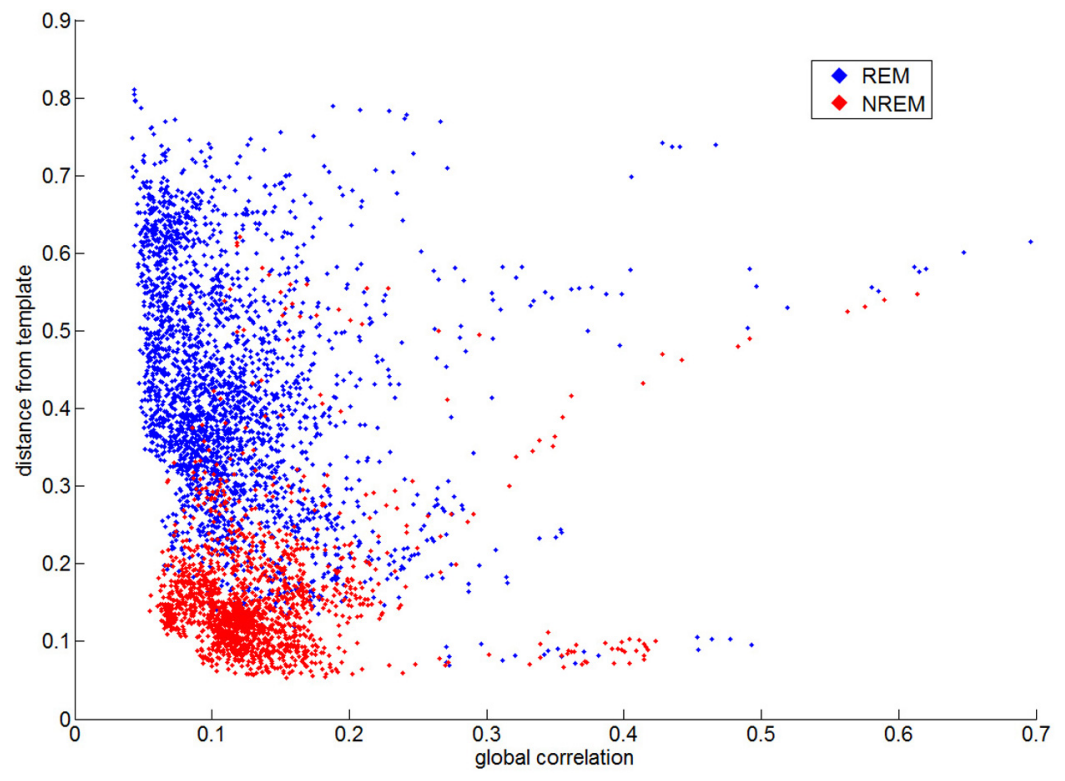

Figure 9. Relationship between global correlations and momentary correlations to sleep template. Strength of global (averaged across all pairs) correlation ( $x$-axis) plotted versus distance from REM/NREM templates accordingly (defined as 1 minus the correlation between the template and the momentary pattern; $y$-axis) for each momentary 5 min correlation structure of either REM sleep (blue) or NREM sleep (red). Data from all subjects plotted together, over 2000 data points for each sleep state in total. Note the striking increase in the range of momentary templates in REM compared with NREM sleep.

Sham tests using correlations between irrelevant time courses while maintaining the inherent structure of the electrodes or permutation tests shuffling electrode locations revealed that this endo-activation phenomenon could not occur by chance. Furthermore, the fact that the similarity between the sleep patterns and the sensory patterns was affected by sleep stages (Figs. 7, 8) also argues against random fluctuations as the source of this phenomenon.

Neither can our results be explained by direct current spread from neighboring electrodes. It has previously been shown that ECoG signals carry a wealth of information sufficient for the decoding of behavioral events even at the subcentimeter level (Wang et al., 2009; Slutzky et al., 2010; Flinker et al., 2011), and that these signals reliably represent the underlying local neural population (Toda et al., 2011). Moreover, in our own data, the most highly correlated electrodes across movie presentations were often not neighbors (Fig. 6). We also used a neighbor removal analysis to test directly whether direct current spread could cause the endo-activation, and found that this was not the case in our data (Fig. 5a).

While the similarity between the sleep patterns and sensory-induced patterns was highly significant, substantial differences were still observed (Figs. 3, 4). This is not surprising; note that the sleep correlation patterns were composed of both sensory and nonsensory networks. In contrast, the crosspresentation analysis used for the movie patterns isolated the sensory-driven networks and prevented spontaneous activity and nonsensory networks from contributing to the awake correlation patterns.

\section{Fine-structured endo-activations}

It should be noted that the highly significant endo-activation we observed could emerge only if the sleep fluctuations were manifesting a specific internal structure and could not be a result of uniform brain fluctuations, since removing such common global signals did not abolish the endo-activation (Fig. 5a). This evidence for a fine correlation structure during sleep is compatible with recent observations of such structure both in human single units and ECoG (Nir et al., 2008). Here we extend this observation by demonstrating that these fine sleep patterns are not random but, rather, reflect, at least partially, the sensory-driven functional organization revealed during the waking state (Figs. 3, 4).

It was difficult to map the correlation patterns directly on the cortex, as our analysis looked at pairs of electrodes, rather than single electrodes. However, this problem was circumvented by plotting the contribution of individual electrodes to the endo-activation (i.e., how much each electrode contributed to the similarity between the sleep pattern and the sensory-evoked pattern). Our analysis revealed that these electrodes were mainly located in well known visual and auditory areas. This result is compatible with the location of the sensory electrodes of the subjects (Fig. 2) and with previous work from our laboratory showing that, in healthy subjects as well, brain areas that respond to the movie (measured by the degree of their reproducibility across presentations, as shown here) were mainly clustered in sensory cortices (Hasson et al., 2004).

The link between the sensory response of each electrode (its reproducibility) and its contribution to endo-activation (Figs. $5 b, 6)$ provides further validation that, although our analysis was limited to measuring responses across electrode pairs, our assessment of endo-activation was sufficiently sensitive to correctly capture the movie-driven functional organization. Our results are also compatible with the previous observation of endo-activation of V1 functional columns obtained in anesthetized cats (Kenet et al., 2003) and extend the observation to human sensory cortex during sleep.

It is important to emphasize that the endo-activation we observed was that of the correlation structure as averaged across time, and not the specific time line of movie responses. In other words, electrodes that tended to comodulate their activity across movie repeats in the waking state also comodulated their activity spontaneously during sleep. However, we have not obtained evidence that the unique chain of activations that occurred in the movie (e.g., activation to faces, followed by tools and then scenery landmarks) was replayed again during sleep. Note also that sensory experience of the specific movie content was not essential for the endo-activation phenomenon, since it occurred also in patients who were not exposed to the movie before falling asleep. A more plausible explanation for the endo-activation is that the naturalistic movie uncovered the underlying functional organization of sensory networks, which, as we show here, tend to activate in a similar fashion both during movie watching and during sleep.

\section{Link to sleep stages}

In the present study, due to limitations imposed by the clinical setting, we were unable to assess the cognitive experience of the subjects during the course of sleep, and hence were unable 
to systematically relate the endo-activation phenomenon to, for example, dreaming and dream content. Note also that sleep in the hospital setting is different and exhibits different staging dynamics than "normal" sleep, and of course includes a far greater number of awakenings (Terzano et al., 1990). This is clearly demonstrated in Figures 7 and 8, which both show atypical sleep hypnograms. Many epileptic patients also suffer from various kinds of sleep disturbances, which might also explain the uncharacteristic sleep structure seen for these nights (Bazil, 2003). Although it is now known that dreams are not limited to REM sleep (Cavallero et al., 1992; Hobson and Pace-Schott, 2002; Suzuki et al., 2004; Nir and Tononi, 2010), the fact that endo-activation epochs were significantly more persistent during NREM periods (Fig. 8) may appear puzzling. Epochs of high endo-activation during NREM sleep may be related to memory consolidation or homeostatic processes, which, as has been previously suggested, may be one of the functions of NREM sleep (Hobson and Pace-Schott, 2002; Tononi and Cirelli, 2006; Nir and Tononi, 2010). Nevertheless, it is important to note that a significant level of endoactivation was also present during REM sleep.

Our analysis of the dynamics of the momentary correlation patterns revealed that the range of momentary patterns was significantly different in the different sleep stages. This is clearly shown in Figure 9, which reveals the larger range of patterns that occur during REM compared with NREM sleep (compare vertical spreads of the blue REM vs red NREM dots).

It could be argued that the larger range of REM sleep patterns was merely due to increased local noise. For example, since high-frequency broadband power was significantly increased during REM sleep, such higher amplitude may have led to increased variance, assuming a simple Poisson process. However, such a simple enhanced noise model predicts a reduction in the averaged pairwise correlations, since the noise is added independently to each electrode and, hence, should tend to decorrelate their activations. As can be seen in Figure 9, contrary to this prediction of local noise, the momentary REM and NREM sleep patterns had similar levels of global (average) correlation levels, as indicated by their overlap along the horizontal axis. Furthermore, we failed to find any correlation between the variability of the sleep patterns (indicated by the distance of each momentary pattern from the mean template) and the level of overall correlation. Together, these results strongly support the notion that the increased range of patterns that occur during REM sleep was indeed an inherent characteristic of the REM stage, which differed from the more stable and repetitive nature of the NREM patterns.

What could be the functional meaning of such a greater repertoire of patterns that emerge during REM sleep? It is tempting to speculate that such increased pattern variability actually reflects a richer space of cognitive states, such as diverse dream contents/types. However, verifying this notion will necessitate subjective reports from the patients, who were not available in the present study.

In summary, REM and NREM sleep have hitherto been characterized mainly by differences in local signals. Here we reveal a new distinction, which is apparent only at the level of network correlations. Additional experiments will be necessary to tease out the exact nature and identity of the different networks that are active during REM sleep, and to test the cognitive counterpart of the tendency of REM to move be- tween different network configurations, compared with the more stable and stereotypical NREM sleep patterns.

\section{Similarity to resting state patterns during the awake state}

Another relevant dataset concerns the phenomena of spontaneous network correlations during awake, resting states. A large body of data has now indicated that in the absence of stimulation or overt tasks, large-scale brain networks spontaneously fluctuate in a coherent manner (Biswal et al., 1995; Nir et al., 2006; Shehzad et al., 2009; Ramot et al., 2011; Yeo et al., 2011) and that these spontaneous fluctuations correspond, at least in part, to well known large-scale functional networks (Keller et al., 2011; Power et al., 2011). Our study now extends this phenomenon to the different stages of sleep. However, the precise relationship of the resting state network correlations to those reported here during sleep remains to be explored. Particularly intriguing is the possibility that the resting state patterns and their dynamic momentary fluctuations may show a differential resemblance to one sleep stage (e.g., NREM sleep) over the other. But these fundamental questions remain to be studied.

\section{Relevance to states of consciousness}

Given the robust and patterned nature of cortical activation during NREM stages, which are likely linked to reduced consciousness, one may wonder what the mechanism could be that blocks conscious processing from emerging under such conditions. At present, the answer to this fundamental question remains unknown. A plausible explanation, in line with previous suggestions (Massimini et al., 2005; Nir et al., 2011), could be related to a prominent characteristic of NREM sleepthe sharp "off" periods, characterized by low gamma power and reduced spiking activity, which are likely reflected in our observation of a global reduction in high-frequency broadband power during NREM sleep. It could be the case that these down, low-broadband power periods, "quench" cortical signal communication (Fries, 2009), and prevent sensory areas from distributing information to memory regions necessary for subjective recollection and reportability upon awakening. At this point, this remains only a plausible conjecture.

While our analysis limits us to mostly sensory areas, evidence regarding other brain regions is emerging from fMRI studies of connectivity during sleep. However, these results are still rather inconsistent. A number of studies have reported reduced connectivity during sleep compared with wakefulness (e.g., in areas of the default mode network; Larson-Prior et al., 2011) or changes in default mode network connectivity between REM and NREM sleep (Koike et al., 2011). Other studies found no such reduction (Horovitz et al., 2008). Another study has found a reduction in connectivity between the thalamus and other cortical sites during light sedation (Gili et al., 2013). It is possible that wakefulness and different sleep stages would also show differences in connectivity patterns between thalamic sites, or within the default mode network, and that this would help explain the differences in levels of consciousness between these states, but these data are not available to us in this present study.

\section{Notes}

Supplemental material for this article is available at http://www. weizmann.ac.il/neurobiology/labs/malach/electrodegroups. HBB-BLP responses of seven electrodes in one patient, shown for the movieviewing sessions and for sleep. The movie shows the division of electrodes 
into "working groups," which tend to coactivate both for an appropriate stimulus during the movie and spontaneously during sleep, regardless of the distance between electrodes. This material has not been peer reviewed.

\section{References}

Andrillon T, Nir Y, Staba RJ, Ferrarelli F, Cirelli C, Tononi G, Fried I (2011) Sleep spindles in humans: insights from intracranial EEG and unit recordings. J Neurosci 31:17821-17834. CrossRef Medline

Bazil CW (2003) Epilepsy and sleep disturbance. Epilepsy Behav 4 [Suppl 2]:S39-S45. CrossRef Medline

Biswal B, Yetkin FZ, Haughton VM, Hyde JS (1995) Functional connectivity in the motor cortex of resting human brain using echo-planar MRI. Magn Reson Med 34:537-541. CrossRef Medline

Canolty RT, Knight RT (2010) The functional role of cross-frequency coupling. Trends Cogn Sci 14:506-515. CrossRef Medline

Cavallero C, Cicogna P, Natale V, Occhionero M, Zito A (1992) Slow wave sleep dreaming. Sleep 15:562-566. Medline

Chauvette S, Volgushev M, Timofeev I (2010) Origin of active states in local neocortical networks during slow sleep oscillation. Cereb Cortex 20: 2660-2674. CrossRef Medline

Contreras D, Steriade M (1995) Cellular basis of EEG slow rhythms: a study of dynamic corticothalamic relationships. J Neurosci 15:604-622. Medline

Crunelli V, Hughes SW (2010) The slow $(<1 \mathrm{~Hz})$ rhythm of non-REM sleep: a dialogue between three cardinal oscillators. Nat Neurosci 13: 9-17. CrossRef Medline

Delorme A, Makeig S (2004) EEGLAB: an open source toolbox for analysis of single-trial EEG dynamics including independent component analysis. J Neurosci Methods 134:9-21. CrossRef Medline

Donner TH, Siegel M (2011) A framework for local cortical oscillation patterns. Trends Cogn Sci 15:191-199. CrossRef Medline

Esser SK, Huber R, Massimini M, Peterson MJ, Ferrarelli F, Tononi G (2006) A direct demonstration of cortical LTP in humans: a combined TMS/EEG study. Brain Res Bull 69:86-94. CrossRef Medline

Fisch L, Privman E, Ramot M, Harel M, Nir Y, Kipervasser S, Andelman F, Neufeld MY, Kramer U, Fried I, Malach R (2009) Neural "ignition": enhanced activation linked to perceptual awareness in human ventral stream visual cortex. Neuron 64:562-574. CrossRef Medline

Flinker A, Chang EF, Barbaro NM, Berger MS, Knight RT (2011) Subcentimeter language organization in the human temporal lobe. Brain Lang 117:103-109. CrossRef Medline

Fries P (2009) Neuronal gamma-band synchronization as a fundamental process in cortical computation. Annu Rev Neurosci 32:209-224. CrossRef Medline

Gili T, Saxena N, Diukova A, Murphy K, Hall JE, Wise RG (2013) The thalamus and brainstem act as key hubs in alterations of human brain network connectivity induced by mild propofol sedation. J Neurosci 33: 4024-4031. CrossRef Medline

Hasson U, Nir Y, Levy I, Fuhrmann G, Malach R (2004) Intersubject synchronization of cortical activity during natural vision. Science 303:16341640. CrossRef Medline

He BJ, Snyder AZ, Zempel JM, Smyth MD, Raichle ME (2008) Electrophysiological correlates of the brain's intrinsic large-scale functional architecture. Proc Natl Acad Sci U S A 105:16039-16044. CrossRef Medline

Hobson JA, Pace-Schott EF (2002) The cognitive neuroscience of sleep: neuronal systems, consciousness and learning. Nat Rev Neurosci 3:679693. CrossRef Medline

Hobson JA, Pace-Schott EF, Stickgold R (2000) Dreaming and the brain: toward a cognitive neuroscience of conscious states. Behav Brain Sci 23: 793-842. Medline

Horovitz SG, Fukunaga M, de Zwart JA, van Gelderen P, Fulton SC, Balkin TJ, Duyn JH (2008) Low frequency BOLD fluctuations during resting wakefulness and light sleep: a simultaneous EEG-fMRI study. Hum Brain Mapp 29:671-682. CrossRef Medline

Huber R, Ghilardi MF, Massimini M, Tononi G (2004) Local sleep and learning. Nature 430:78-81. CrossRef Medline

Huber R, Ghilardi MF, Massimini M, Ferrarelli F, Riedner BA, Peterson MJ, Tononi G (2006) Arm immobilization causes cortical plastic changes and locally decreases sleep slow wave activity. Nat Neurosci 9:1169-1176. CrossRef Medline

Ji D, Wilson MA (2007) Coordinated memory replay in the visual cortex and hippocampus during sleep. Nat Neurosci 10:100-107. CrossRef Medline

Kang K, Shelley M, Henrie JA, Shapley R (2010) LFP spectral peaks in V1 cortex: network resonance and cortico-cortical feedback. J Comput Neurosci 29:495-507. CrossRef Medline

Keller CJ, Bickel S, Entz L, Ulbert I, Milham MP, Kelly C, Mehta AD (2011) Intrinsic functional architecture predicts electrically evoked responses in the human brain. Proc Natl Acad Sci U S A 108:10308-10313. CrossRef Medline

Kenet T, Bibitchkov D, Tsodyks M, Grinvald A, Arieli A (2003) Spontaneously emerging cortical representations of visual attributes. Nature 425 : 954-956. CrossRef Medline

Koike T, Kan S, Misaki M, Miyauchi S (2011) Connectivity pattern changes in default-mode network with deep non-REM and REM sleep. Neurosci Res 69:322-330. CrossRef Medline

Lachaux JP, George N, Tallon-Baudry C, Martinerie J, Hugueville L, Minotti L, Kahane P, Renault B (2005) The many faces of the gamma band response to complex visual stimuli. Neuroimage 25:491-501. CrossRef Medline

Larson-Prior LJ, Power JD, Vincent JL, Nolan TS, Coalson RS, Zempel J, Snyder AZ, Schlaggar BL, Raichle ME, Petersen SE (2011) Modulation of the brain's functional network architecture in the transition from wake to sleep. Prog Brain Res 193:277-294. CrossRef Medline

Massimini M, Ferrarelli F, Huber R, Esser SK, Singh H, Tononi G (2005) Breakdown of cortical effective connectivity during sleep. Science 309: 2228-2232. CrossRef Medline

Mukamel R, Gelbard H, Arieli A, Hasson U, Fried I, Malach R (2005) Coupling between neuronal firing, field potentials, and FMRI in human auditory cortex. Science 309:951-954. CrossRef Medline

Mukamel R, Nir Y, Harel M, Arieli A, Malach R, Fried I (2011) Invariance of firing rate and field potential dynamics to stimulus modulation rate in human auditory cortex. Hum Brain Mapp 32:1181-1193. CrossRef Medline

Nir Y, Tononi G (2010) Dreaming and the brain: from phenomenology to neurophysiology. Trends Cogn Sci 14:88-100. CrossRef Medline

Nir Y, Hasson U, Levy I, Yeshurun Y, Malach R (2006) Widespread functional connectivity and fMRI fluctuations in human visual cortex in the absence of visual stimulation. Neuroimage 30:1313-1324. CrossRef Medline

Nir Y, Fisch L, Mukamel R, Gelbard-Sagiv H, Arieli A, Fried I, Malach R (2007) Coupling between neuronal firing rate, gamma LFP, and BOLD fMRI is related to interneuronal correlations. Curr Biol 17:1275-1285. CrossRef Medline

Nir Y, Mukamel R, Dinstein I, Privman E, Harel M, Fisch L, Gelbard-Sagiv H, Kipervasser S, Andelman F, Neufeld MY, Kramer U, Arieli A, Fried I, Malach R (2008) Interhemispheric correlations of slow spontaneous neuronal fluctuations revealed in human sensory cortex. Nat Neurosci 11:1100-1108. CrossRef Medline

Nir Y, Staba RJ, Andrillon T, Vyazovskiy VV, Cirelli C, Fried I, Tononi G (2011) Regional slow waves and spindles in human sleep. Neuron 70: 153-169. CrossRef Medline

Power JD, Cohen AL, Nelson SM, Wig GS, Barnes KA, Church JA, Vogel AC, Laumann TO, Miezin FM, Schlaggar BL, Petersen SE (2011) Functional network organization of the human brain. Neuron 72:665-678. CrossRef Medline

Privman E, Fisch L, Neufeld MY, Kramer U, Kipervasser S, Andelman F, Yeshurun Y, Fried I, Malach R (2011) Antagonistic relationship between gamma power and visual evoked potentials revealed in human visual cortex. Cereb Cortex 21:616-624. CrossRef Medline

Privman E, Malach R, Yeshurun Y (2013) Modeling the electrical field created by mass neural activity. Neural Netw 40:44-51. CrossRef Medline

Ramot M, Wilf M, Goldberg H, Weiss T, Deouell LY, Malach R (2011) Coupling between spontaneous (resting state) fMRI fluctuations and human oculo-motor activity. Neuroimage 58:213-225. CrossRef Medline

Sederberg PB, Kahana MJ, Howard MW, Donner EJ, Madsen JR (2003) Theta and gamma oscillations during encoding predict subsequent recall. J Neurosci 23:10809-10814. Medline

Shehzad Z, Kelly AM, Reiss PT, Gee DG, Gotimer K, Uddin LQ, Lee SH, Margulies DS, Roy AK, Biswal BB, Petkova E, Castellanos FX, Milham MP (2009) The resting brain: unconstrained yet reliable. Cereb Cortex 19: 2209-2229. CrossRef Medline

Slutzky MW, Jordan LR, Krieg T, Chen M, Mogul DJ, Miller LE (2010) 
Optimal spacing of surface electrode arrays for brain-machine interface applications. J Neural Eng 7:26004. CrossRef Medline

Staba RJ, Wilson CL, Fried I, Engel J Jr (2002) Single neuron burst firing in the human hippocampus during sleep. Hippocampus 12:724-734. CrossRef Medline

Steriade M, McCormick DA, Sejnowski TJ (1993) Thalamocortical oscillations in the sleeping and aroused brain. Science 262:679-685. CrossRef Medline

Steriade M, Timofeev I, Grenier F (2001) Natural waking and sleep states: a view from inside neocortical neurons. J Neurophysiol 85:1969-1985. Medline

Suzuki H, Uchiyama M, Tagaya H, Ozaki A, Kuriyama K, Aritake S, Shibui K, Tan X, Kamei Y, Kuga R (2004) Dreaming during non-rapid eye movement sleep in the absence of prior rapid eye movement sleep. Sleep 27: 1486-1490. Medline

Talairach J, Tournoux P (1988) Co-planar stereotaxic atlas of the human brain. New York: Thieme Medical.

Terzano MG, Parrino L, Fioriti G, Orofiamma B, Depoortere H (1990) Modifications of sleep structure induced by increasing levels of acoustic perturbation in normal subjects. Electroencephalogr Clin Neurophysiol 76:29-38. CrossRef Medline
Toda H, Suzuki T, Sawahata H, Majima K, Kamitani Y, Hasegawa I (2011) Simultaneous recording of ECoG and intracortical neuronal activity using a flexible multichannel electrode-mesh in visual cortex. Neuroimage 54:203-212. CrossRef Medline

Tononi G, Cirelli C (2006) Sleep function and synaptic homeostasis. Sleep Med Rev 10:49-62. CrossRef Medline

Volgushev M, Chauvette S, Mukovski M, Timofeev I (2006) Precise long-range synchronization of activity and silence in neocortical neurons during slow-wave oscillations [corrected]. J Neurosci 26:56655672. CrossRef Medline

Wang W, Degenhart AD, Collinger JL, Vinjamuri R, Sudre GP, Adelson PD, Holder DL, Leuthardt EC, Moran DW, Boninger ML, Schwartz AB, Crammond DJ, Tyler-Kabara EC, Weber DJ (2009) Human motor cortical activity recorded with Micro-ECoG electrodes, during individual finger movements. Conf Proc IEEE Eng Med Biol Soc 2009: 586-589. CrossRef Medline

Yeo BT, Krienen FM, Sepulcre J, Sabuncu MR, Lashkari D, Hollinshead M, Roffman JL, Smoller JW, Zöllei L, Polimeni JR, Fischl B, Liu H, Buckner RL (2011) The organization of the human cerebral cortex estimated by intrinsic functional connectivity. J Neurophysiol 106: 1125-1165. CrossRef Medline 